\title{
LEAD: a linguistic approach to dynamic adaptability for practical applications
}

N. Amano and T. Watanabe

Graduate School of Information Science, Japan Advanced Institute of Science and Technology (JAIST)

1-1 Asahidai, Tatsunokuchi, Ishikawa, 923-12, Japan

Tel: +81-761-51-1256 Fax: +81-761-51-1149

E-Mail: \{n-amano, takuo\}@jaist.ac.jp

\begin{abstract}
A system has dynamic adaptability if it adapts itself to a changing runtime environment. As open-ended distributed systems and mobile computing systems have spread widely, the need for software with dynamic adaptability increases. We designed and implemented a language LEAD that provides an architecture for dynamic adaptability. The basic idea is to introduce a mechanism that affects procedure invocation dynamically according to the runtime environment. Using LEAD, we can easily achieve 1) construction of highly extensible dynamically adaptable applications, and 2) addition of dynamic adaptability into existing applications.
\end{abstract}

\section{Keywords}

Software adaptation, meta-level architecture, reflection, mobile computing

\section{INTRODUCTION}

Open-ended distributed systems and/or mobile computing systems are becoming common. When designing applications for such systems, accurate advance information about its runtime environment is rarely obtainable. For example, the logical/physical location, hardware configurations (esp. including PC-cards), etc., may be unknown. Moreover, mobile agents in Java[1], Telescript[2] etc., can move between computing sites via the network. All elements (kind of computer, hardware and software configurations, physical surroundings of computer, etc.) may change. We use the phrase runtime environment to express these elements. Dynamic changes in runtime environments have a strong influence on application execution. We cannot ignore them.

For example, 1) we use an application using remote procedure call (RPC) in mobile computing systems, 2) we use a full WWW browser on a personal dig- 
ital assistant (PDA). In the first case, when the network connectivity changes from wired to wireless, and vice versa, a problem occurs. Running the application connected to the network for a long time does not go well, because of low bandwidth, and possible disconnection of wireless communication, etc. In this case, we can handle the problem effectively by changing the behavior of the application from RPC to remote programming (RP) form [2]. In the second case, since the CPU power of a typical PDA is poor, and the hardware resources have many limitations, use of a large application like a full WWW browser is difficult. In this case, we can use it comfortably by restricting some functionalities. For example, by reducing the quality of images, or eliminating images, etc. Moreover, as the display size of typical PDAs is small, if we use the browser used on desktop environments, the look and feel becomes poor. We can avoid this by changing the sizes of fonts, windows and widgets, and the layouts of widgets, etc.

It is difficult to implement such specific applications with adaptability to the runtime environment individually, because we cannot anticipate the dynamic changes in advance. The cost of the implementation is too high. Therefore, applications must have the ability to adapt themselves to dynamic changes. Such applications can make full use of properties in runtime environments and can handle limitations in them. We call such an ability dynamic adaptability; applications which have it are dynamically adaptable applications (DAAs).

To realize dynamic adaptability, we need both operating system (OS) level adaptation and application level adaptability. The OS realizes the former through Plug and Play devices, dynamic alteration of IP addresses, etc. The latter occurs when applications change their behavior according to dynamic changes in runtime environments. Until now, only adaptation at the OS-level has been investigated. These OSs are called adaptable OSs. There are SPIN[3], Exokernel[4], Apertos[5], etc. However, that alone is insufficient. To realize dynamic adaptability, we need to change the application's behavior. The relation between both kinds of adaptation is complementary. We aim for effective realization of adaptation at the application level. Our main goals are the following: 1) the realization of highly extensible DAAs, 2) the introduction of dynamic adaptability into applications in existing languages, 3) the separation of dynamic adaptability from the primary subject domain in the applications. The effective software architecture which satisfies these points is the meta-level architecture [6, 7]. A DAA in LEAD, our proposed language, forms a metalevel architecture, and has reflectivity[6]. Using LEAD, we can easily realize our main goals.

The remainder of this paper is organized as follows. Section 2 describes the required mechanism to realize dynamic adaptability. Section 3 gives an overview of the architecture of DAAs in LEAD. Section 4 covers the main functionalities of LEAD. Section 5 discusses the runtime environment of LEAD. Section 6 shows programming in LEAD by using examples. Section 7 compares LEAD with related work. Finally, Section 8 provides a conclusion. 


\section{DYNAMIC ADAPTABILITY}

To realize the dynamic adaptability, we have designed a mechanism which changes procedure invocation dynamically according to the state of the runtime environments. In this mechanism, there are several definitions for a procedure in an application. Then, the mechanism selects a suitable definition depending on the state, and it makes the application execute the selected definition (procedure). This mechanism has following merits. 1) The control mechanism of dynamic adaptability becomes simple, reducing the cost. 2) By adding suitable definitions depending on the states of runtime environments to the procedures, the extensibility of DAAs is improved. 3) This mechanism is well-suited to existing languages which have procedure call. Thus, we can easily apply this mechanism to applications written in existing languages. We call the procedures which change according to the states of runtime environments generic procedures, and call the definitions of the procedures methods. To realize generic procedures effectively, the following mechanisms are necessary.

1. The mechanism which obtains the states of environment elements (e-elements) that construct the runtime environments.

2. The mechanism which defines highly abstract e-elements and their states.

3. The mechanism to describe a pair of runtime environment states and a suitable method depending on the state as an independent module from application programs.

4. The mechanism which selects a suitable method depending on a state of runtime environments (adaptation mechanism).

Since a runtime environment consists of several states of e-elements, the second mechanism is required. This mechanism hides low level states in the runtime environment from application programmers, therefore they can concisely describe highly readable programs.

If we describe the codes of dynamic adaptability (adaptation codes) in applications directly, the adaptation codes and the codes of primary subject domain (primary codes) in the applications are mixed. Thus, readability of programs is reduced, and their maintenance becomes harder. Moreover, since it is difficult to handle unexpected states beforehand, extensibility of programs also becomes harder. These problems can be solved by the third mechanism (Fig.1). We can also realize reusing and/or sharing of adaptation codes easily by the third mechanism.

To realize a generic procedure, we must prepare several suitable methods depending on the states of runtime environments. However, in open-ended systems and/or mobile computing systems, we cannot prepare all methods which correspond to all states. Therefore, we need the fourth mechanism and the strategy which links the states with the methods (adaptation strategy). To 
realize dynamic adaptability in a flexible way, the adaptation strategy must be programmable. We also need a mechanism to describe it.

LEAD is a language which has the above mechanisms. The LEAD runtime system (LRS) works as a server which provides dynamic adaptability for applications.

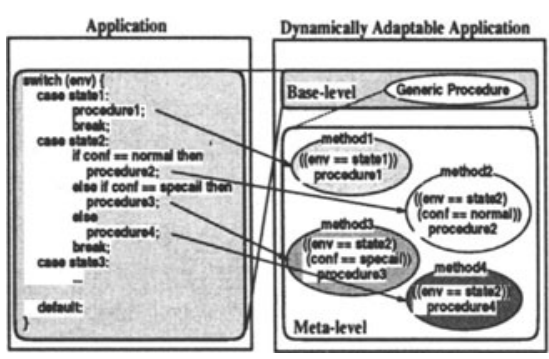

Figure 1 Separation of concerns

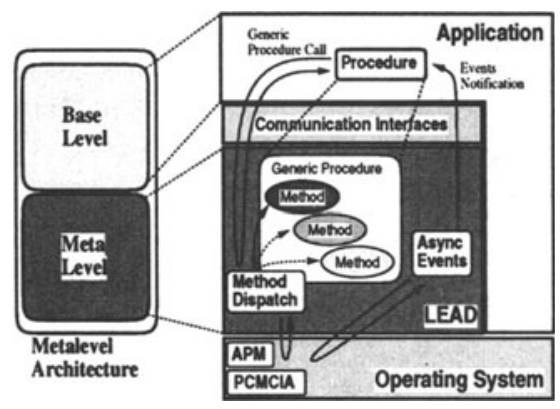

Figure 2 A dynamically adaptable application

\section{THE ARCHITECTURE OF DAAS IN LEAD}

A DAA in LEAD forms a meta-level architecture, consisting of an application (base-level) and LRS (meta-level) (Figure 2). Base level procedures should be changed according to the states of runtime environments They are defined as generic procedures and meta level methods using LEAD. The runtime environment of LEAD provides interfaces to communicate at both levels. The base level calls a generic procedure at the meta level via the interfaces. When the meta level receives a generic procedure call request, it obtains runtime environment state information from the OS. Then, using a built-in adaptation strategy, it selects a suitable method. The selected method is sent to the base level via the interface and is executed there. These actions are combined into a generic procedure call.

It is difficult to realize DAAs which regard state changes as triggers, and change their own behavior by generic procedure call only. LEAD has asynchronous event functionality where the events express the occurrence of the states' changes of e-elements linked with the events' names. Using this functionality, we can implement DAAs efficiently and concisely. Asynchronous events are used with generic procedures which define the actions linked with occurrences of events. Applications can use events by specifying the events' names and generic procedures for the events in LRS via the interfaces. When the states of e-elements (events) which the application specified change, LRS notifies the application of changes asynchronously. In the application, the 
generic procedures linked with the events are called (executed) automatically. These actions are combined into the specifying events' names and generic procedures for the events. Moreover, we can achieve event filtering easily and implicitly by using generic procedures as actions for the events.

\section{MAIN FUNCTIONALITIES OF LEAD}

\subsection{Built-in functions for e-elements}

LEAD has built-in functions to obtain the states of e-elements (Table 1). These built-in functions' names are linked with the e-elements' names. Each function returns the state of the linked e-element as a numerical value.

Table 1 Some built-in functions for hardware configuration

\begin{tabular}{ll}
\hline Built-in Functions (e-elements) & Return Value (state of e-elements) \\
\hline network & network connectivity \\
memory & free memory size \\
display & display size \\
power & power source type \\
battery & battery remainder \\
\hline
\end{tabular}

\subsection{Definition of e-elements and states}

The abstraction of these e-elements (built-in functions) and their states (return value) is low, and runtime environments consist of several e-elements. Thus we define higher level abstract e-elements and their states by using several e-elements. The following pseudocode defines more abstract e-element emergency and its states (TRUE, FALSE) by using memory and battery.

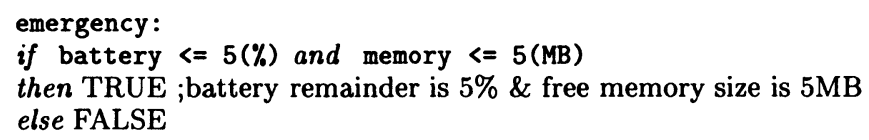

A new higher level abstract e-element such as emergency can be shared and/or reused among several DAAs.

\subsection{Generic procedures and methods}

Generic procedures consist of elements in Figure 3. When we specify Generic as the $A p p$, the generic procedure can be shared among several DAAs written 
in the same language. The $E$-list is a list of e-elements which are used when the LRS selects a suitable method depending on the state of runtime environments from several methods which belong to the generic procedure.

\begin{tabular}{lll}
\hline$\langle$ Generic Procedure $\rangle$ & $::=$ & $(\langle$ Name $\rangle\langle$ Lang $\rangle\langle$ App $\rangle\langle$ E-list $\rangle)$ \\
\hline$\langle$ Name $\rangle$ & $::=$ & $\langle$ Generic procedure's name $\rangle$ \\
$\langle$ Lang $\rangle$ & $::=$ & $\langle$ Application's description language name $\rangle$ \\
$\langle$ App $\rangle$ & $::=$ & $\langle$ Application's name $\rangle \mid$ Generic \\
$\langle E$-list $\rangle$ & $::=$ & $\left(\{\langle\text { e-element }\rangle\}^{*}\right)$ \\
\hline
\end{tabular}

Figure 3 The construction elements of generic procedures

Methods consist of elements in Figure 4. The $S$-list is a list that describes the states of e-elements which can be selected, namely it is a list of conditions for eelements defined in a generic procedure. Each condition in a $S$-list is evaluated at runtime by LRS. The evaluation is based on an adaptation strategy of LRS. A suitable method depending on the state of runtime environments is selected according to the result of this evaluation.

The Location specifies the location of the host where the selected method is actually executed. We can use remote execution of methods, when we change $\mathrm{RPC}$ form into RP form, and vice versa, depending on network connectivity. (See Section 1.) We describe the execution code of the method (actual behavior) in the App-Proc. There are two ways to define App-Proc as follows. We describe, 1) the procedure's code as a string, or 2) the name of the procedure which implements the actual behavior in the application.

\begin{tabular}{lll}
\hline$\langle$ Method $\rangle$ & $::=$ & $(\langle$ Name $\rangle\langle$ Lang $\rangle\langle$ App $\rangle\langle$ S-list $\rangle\langle$ Location $\rangle\langle$ App-Proc $\rangle)$ \\
\hline$\langle$ Name $\rangle$ & $::=$ & (Generic procedure's name $\rangle$ \\
$\langle$ Lang $\rangle$ & $::=$ & AApplication's description language name $\rangle$ \\
$\langle$ App $\rangle$ & $::=$ & (Application's name $\rangle \mid$ Generic \\
$\langle$ S-list $\rangle$ & $::=$ & $\left(\{\langle\text { state of e-element }\rangle\}^{*}\right)$ \\
$\langle$ Location $\rangle$ & $::=$ & $\langle$ Host name $\rangle \mid$ local $\mid$ remote \\
$\langle$ App-Proc $\rangle$ & $::=$ & $\langle$ Procedure's code $\rangle \mid\langle$ Procedure's name $\rangle$ \\
\hline
\end{tabular}

Figure 4 The construction elements of a method 


\subsection{The definition of adaptation strategy}

Method selection is realized by using the $S$-list in each method and adaptation strategy which LRS provides. The adaptation strategy evaluates the $S$-list in each method, and selects a suitable method depending on the states of runtime environments.

It is impossible to realize a general and/or fixed method of evaluation, because it is affected by the number, the relation, etc. of e-elements which are used in the $S$-list. In LRS, the adaptation strategy is realized as a generic procedure in LEAD. We can customize it easily. Since the adaptation strategy provided by LRS is completely independent of DAAs and their generic procedures, even if we change the strategy, we need not modify existing generic procedures and their methods.

DAAs in LEAD can dynamically alter the adaptation strategy in LRS via the interface. Since the alteration has an influence on the method selection, it reflects the application's behavior. Namely, DAAs in LEAD have reflectivity.

\section{THE RUNTIME ENVIRONMENT OF LEAD}

\subsection{Built-in adaptation strategies}

The built-in adaptation strategies which LRS provides are simple strategy and priority number strategy (priority strategy).

\section{(a) Simple strategy}

The AND strategy and OR strategy are simple strategies. The former evaluates all conditions in the $S$-list by using AND $(\wedge)$, and the latter evaluates them by using OR $(V)$. The simple strategy is easy to understand and implement. However, there are cases where it cannot select a method.

\section{(b) Priority strategy}

In the priority strategy, each e-element is assigned a priority number (Table

2) based on the order in which e-elements are defined in a generic procedure (from left to right).

Table 2 Priority number and priority number list

\begin{tabular}{ll}
\hline number, position, priority number of e-element & $=n, k, 2^{n-k}$ \\
list of e-elements & $=\left(E_{1} \ldots E_{n}\right)$ \\
priority number list (association list) & $=\left(\left(E_{1} \cdot 2^{n-1}\right) \ldots\left(E_{n} \cdot 2^{0}\right)\right)$ \\
\hline
\end{tabular}


When a generic procedure is defined in LRS, LRS generates the priority number list for the generic procedure (Table 2). This list is a set of pairs, which consist of an e-element and its priority number. When LRS evaluates each state (condition) in the $S$-list in turn, LRS totals the priority number of the e-elements whose conditions are false as the negative sum, and are true as the positive sum, respectively (Table 3 ). $\varepsilon$ is an evaluation function on states: if $S_{i}$ is true then $\varepsilon\left(S_{i}\right)=1$, otherwise $\varepsilon\left(S_{i}\right)=0$. Then, a suitable method is decided by comparing these sums among methods. For the comparison, the following rules are applied in turn: $\alpha$ ) to select the method where negative sum is minimum; $\beta$ ) to select the method where positive sum is maximum.

LRS applies the $\alpha$ rule to methods first. If a method cannot be decided, LRS tries to apply the $\beta$ rule to methods selected by the $\alpha$ rule, and to select a method. To definitely select a method, LRS assigns $2^{n}$ as the priority number to each e-element. The priority strategy can always determine a method. This strategy is based on an assumption that programmers specify e-elements in priority order when they define a generic procedure.

Table 3 Positive sum and negative sum of priority numbers

\begin{tabular}{ll}
\hline (e-element, state, priority number) & $\left(E_{1}, S_{1}, 2^{n-1}\right) \cdots\left(E_{n}, S_{n}, 2^{0}\right)$ \\
evaluation function on states & $\varepsilon:$ state $\rightarrow\{0 \mid 1\}$ \\
positive sum & $\sum_{i=1}^{n} \varepsilon\left(S_{i}\right) \cdot 2^{n-i}$ \\
negative sum & $\sum_{i=1}^{n}\left(1-\varepsilon\left(S_{i}\right)\right) \cdot 2^{n-i}$ \\
\hline
\end{tabular}

\subsection{Communication interfaces}

In the LEAD runtime environment, there are interface functions which realize the communication of applications and LRS (Table 4). Applications can utilize functionalities of LRS with these functions. These functions can be executed such as function call in applications. Implementation of these functions depends on the descriptive languages of applications. Therefore, each function is implemented in each language.

Table 4 Interface functions

\begin{tabular}{ll}
\hline Interface Functions & Uses \\
\hline GenericProc & call generic procedure \\
RegistEvent & specify the asynchronous events \\
EventProc & specify the generic procedures for asynchronous events \\
GenericStrategy & change the adaptation strategy \\
\hline
\end{tabular}




\section{PROGRAMMING IN LEAD}

We have implemented a prototype of LEAD as an interpretive language which is an extension of Scheme*. This prototype supports Emacs Lisp, Perl, Tcl/Tk and Python as descriptive languages of DAAs. We can also describe DAAs in LEAD only. Then, we have also implemented the prototype, the communication interfaces for these languages and examples on SunOS 4.1.4 and BSD/OS 2.0.1 with Wildboar (support for APM (advanced power management), PC card services, etc.).

\subsection{Specification of examples}

We have implemented a dynamically adaptable text editor and resource monitor as examples of DAAs (Fig.5,6). These examples are GUI-based applications, and their base language is $T c l / T k$. They can be executed not only on desk-top environments, but also in notebook environments which have PC card slots and are battery-powered. In this paper, we show the description of the editor selectively. The specification of dynamically adaptable behavior in this editor is as follows. 1) It changes the size of the main and dialog windows and font according to the display size. It also changes the size and layout of widgets (improveming the look and feel). 2) It expands/contracts the size of the input-output ( $\mathrm{I} / \mathrm{O})$ buffer dynamically according to free memory size (saving memory and realizing efficient file IO). 3) It dynamically increases/decreases file auto-save intervals according to the remaining battery life.

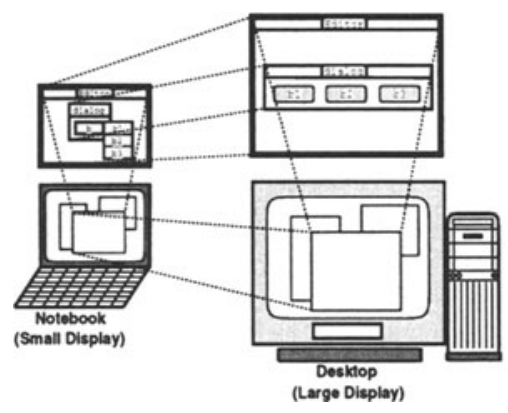

Figure 5 A dynamically adaptable text editor

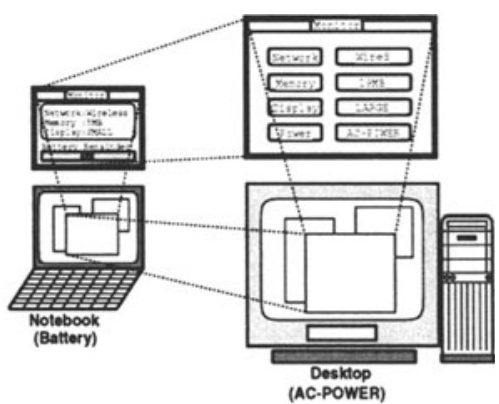

Figure 6 A dynamically adaptable resource monitor

\footnotetext{
${ }^{*}$ It is not essential that the prototype be based on Scheme. We can also realize LEAD with other languages.
} 


\subsection{Describing generic procedures and methods}

Generic procedures are defined by using defgeneric, and methods are defined by using defmethod. These are special forms of LEAD. The following code is part of the generic procedure and its methods which realize the file-selection dialog in the editor.

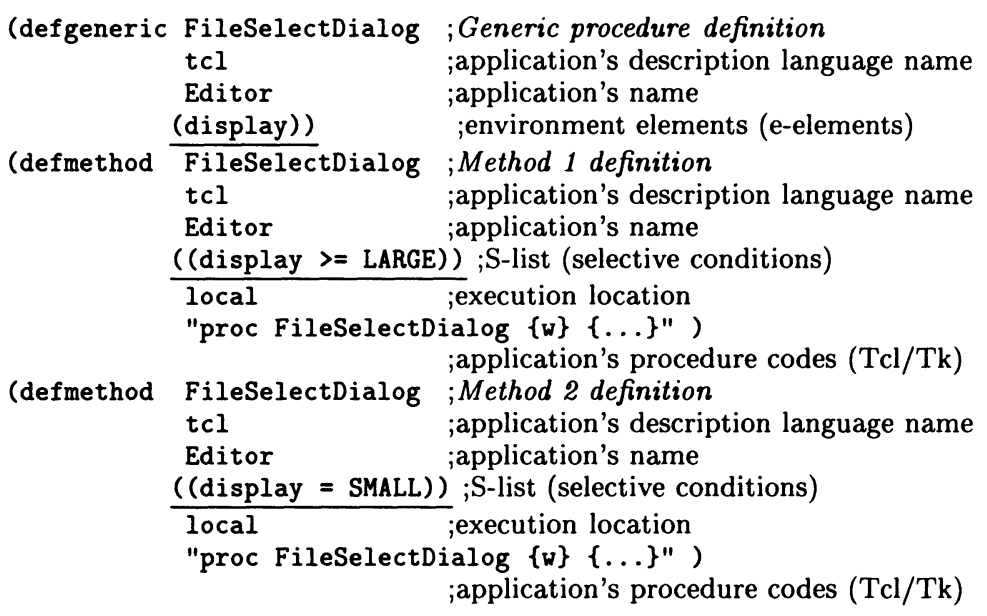

This editor uses display as an e-element to change its own behavior according to the display size. In each method, the condition (state) for display is specified. In above codes, method 1 is executed when the display size is large, and realizes the suitable dialog and widgets for the large display. Method 2 also realizes suitable dialog and widgets for a small display. We describe generic procedure call in the application (editor) as follows.

GenericProc "Editor FileSelectDialog \$w" \#generic procedure call

The arguments of the interface function GenericProc are the application's name, the generic procedure's name and arguments for the generic procedure.

\subsection{Using asynchronous events}

This editor uses an asynchronous event to note a change in the power source (power event). In notebook environments, users may switch the power source from AC power to battery, and vice versa. Changes cannot be predicted. Using asynchronous events, the editor need not poll to discover a change. Thus, its implementation becomes efficient and short. The following code is part of the generic procedure and its methods which realize file auto-save in the editor. 


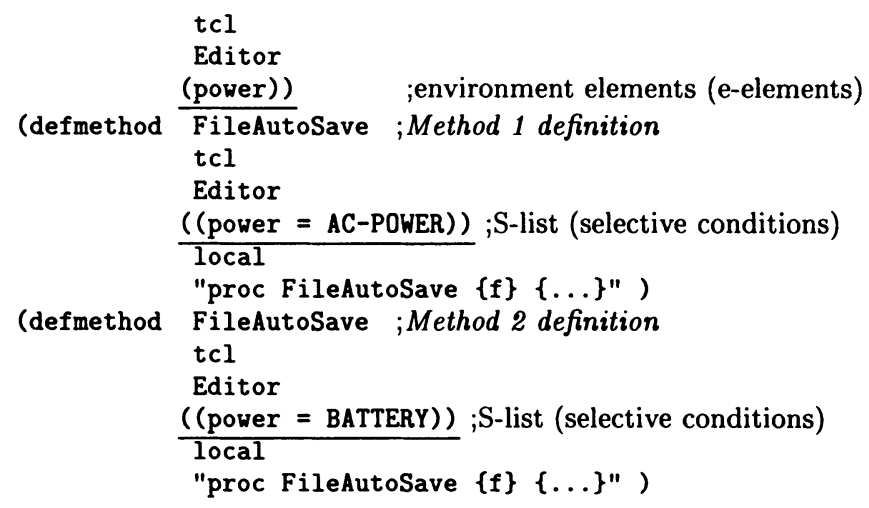

This editor uses power as an e-element to change its behavior according to the power source. In the above code, method 1 is executed if the power source is AC power; if it is battery, method 2 is executed. When the power source is battery, the editor reduces the frequency of file auto-saves and saves battery consumption. To realize this behavior, we wrote the following description in the application (editor).

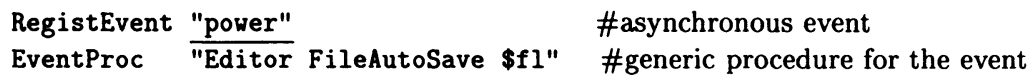

RegistEvent and EventProc are interface functions. We use the former to specify the power event to LRS, and the latter to specify the generic procedure for the event to LRS. For the above editor code, when the power source changes, the editor receives the event asynchronously from LRS and then the specified generic procedure (FileAutoSave) is executed.

\subsection{Event filtering and dynamic adaptation using reflectivity}

In Section 6.3, we showed that the editor varied the frequency of file auto-saves according to the power source. However, we can consider the requirement that if the power source is battery and the battery remainder is about half, the editor decreases the frequency. Then, if the remainder is small, it does not do the file auto-save. We can easily realize this requirement by adding battery as an e-element, and the condition for battery to the generic procedure and method 2 as follows.

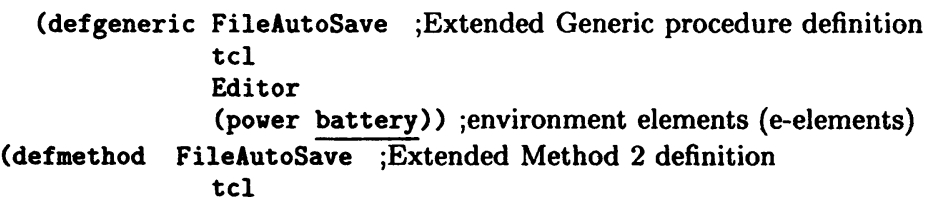




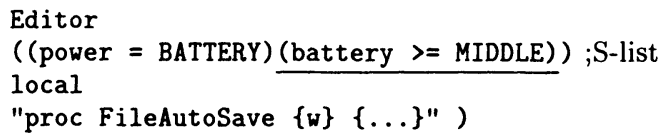

We must also add the battery event to the description in the application (editor) to obtain an occurrence of the change as follows.

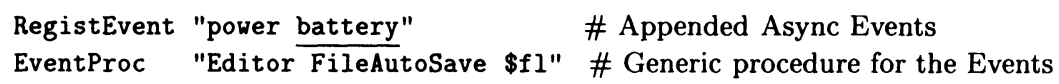

The default adaptation strategy in LRS is the priority strategy. However, in the above case, the editor must not execute any methods for file auto-save if the method which satisfies its conditions in the $S$-list does not exist. Therefore, the priority strategy is unsuitable. To change adaptation strategy, we use the interface function GenericStrategy in the application (editor) as follows.

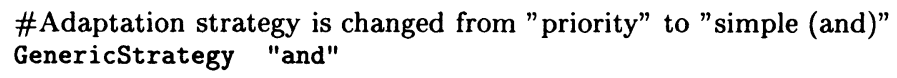

Changing the adaptation strategy from the priority strategy to AND strat$e g y$, if the power source is battery and the remainder is less than MIDDLE, the file auto-save is not executed. Namely, the event is filtered, and the functionality of the editor is restricted according to the state of runtime environments. By changing the adaptation strategy again from the AND strategy to the priority strategy, the restriction is released. We can realize dynamic adaptability in a flexible way as in this case. Changing the adaptation strategy is the same as changing the state in LRS. The result of the alteration reflects the applications' behavior. DAAs in LEAD have such reflectivity. Dynamic adaptability based on alteration of the adaptation strategy uses the reflectivity of DAAs in LEAD.

\section{RELATED WORK}

Most existing work based on a linguistic approach to software adaptation aims at adaptation of objects in higher abstract runtime environments. In compositional interface[8], we need to describe the operations for objects according to the environment in objects completely beforehand. In Rome[9] and Clovers[10], though they can alter objects dynamically, they have not limited the dynamic alteration. Therefore, maintenance of programs becomes harder. In compositional adaptation[11], the objects can be changed dynamically according to the environment. However, it cannot be considered the dynamic change of the environment itself. Gaea[12] does not have a mechanism to express environments explicitly. In AL-1/D[13], meta objects have statistical information about the environment, and methods of meta objects can be executed according to the information. However, the kind of information is fixed 
by the system, and the variety is low. Moreover, abstraction of the information is less.

In LEAD, the $S$-list in a method expresses the states of runtime environments. In a sense, we need part of the description of the runtime environments. However, since the method can be selected by using the $S$-list and the adaptation strategy, we need not to describe the states of runtime environments completely beforehand. Even if the description is incomplete, by using the adaptation strategy, a suitable method depending on the states can be selected. We can minimize the description according to the implementation of the adaptation strategy. Then, defining the higher abstract e-elements, LEAD can realize the adaptation for higher abstract environments such as those in existing works. Moreover, though LEAD can introduce dynamic adaptability into applications written in existing languages, those existing works cannot do it.

\section{CONCLUDING REMARKS}

We have designed an architecture for dynamically adaptable software and implemented its description language LEAD. Moreover, by describing examples in LEAD, we have inspected the effectiveness of LEAD.

Since DAAs in LEAD form a meta-level architecture, the readability is higher and maintenance becomes easier. Generic procedures have higher extensibility and reusability. Using LEAD, we can extend existing software effectively and efficiently.

For future work, we plan to support Java and Telescript. Mobile agents can be described in them without considering the state of the runtime environment. Therefore, they do not work well in some environments. We think that we can solve this problem by applying our approach to these languages.

\section{ACKNOWLEDGMENTS}

We are grateful to Prof. Tatsuo Nakajima and Dr. Kazuhiro Ogata at JAIST. We also thank the members of the language design laboratory at JAIST for discussions and comments. This work is supported in part by IPA (Information technology Promotion Agency, Japan) Advanced Information Technology Program.

\section{REFERENCES}

[1] Gosling, J., Joy, B. and Steele, G.: The Java Language Specification, The Java Series, Addison-wesley publishing company (1996).

[2] White, J. E.: Telescript Technology - Mobile Agents (1996). 
[3] Bershad, B. N., Savage, S., Pardyak, P. and Sirer, E. G.: Extensibility, Safety and Performance in the SPIN Operating System, in Proceedings of the SOSP'95, pp. 267-284 (1995).

[4] Engler, D. R., Kaashoek, M. F. and Jr, J. O.: Exokernel: An Operating System Architecture for Application-Level Resource Management, in Proceedings of the SOSP'95 (1995).

[5] Yokote, Y.: The Apertos Reflective Operating System: The Concept and Its Implementation, in Proceedings of the OOPSLA'92, pp. 414-434 (1992).

[6] Maes, P. and Naddi, D. eds.: Meta-level Architecture and Reflection, ELSEVIER SCIENCE PUBLISHERS B.V. (1988).

[7] Kiczales, G., Rivieres, des J. and Bobrow, D. G.: The Art of the Metaobject Protocol, The MIT Press (1991).

[8] Raj, R. K. and Levy, H. M.: A Compositional Model for Software Reuse, in Proceedings of the ECOOP'89 (1989).

[9] Carre, B., Dekker, L. and Geib, J.: Multiple and Evolutive Representation in the ROME Language, in Proceedings of the TOOLS'90 (1990).

[10] Stein, L. A. and Zdonik, S. B.: Clovers: The Dynamic Behavior of Tyoes and Instances, Technical Report CS-89-42, Department of Computer Science, Brown University (1989).

[11] Honda, Y., Watari, S., Osawa, E. and Tokoro, M.: Compositional Adaptation: A New Method for Constructing Software in Open-Ended Systems, Technical Report SCSL-TR-90-012, Sony CSL Inc (1991).

[12] Nakashima, H.: Organic Programming for Cooperative Computation, in Proceedings of the WWCA'97, pp. B-1-3-1-8 (1997).

[13] Okamura, H., Ishikawa, Y. and Tokoro, M.: AL-1/D: A Distributed Programming System with Multi-Model Reflection Framework, in Proceedings of the IMSA'92 (1992).

\section{BIOGRAPHIES}

Noriki AMANO is a Ph.D. student at the Japan Advanced Institute of Science and Technology (JAIST). His research interests include software adaptation, computational reflection, meta-level architecture and mobile computing. He is a member of JSSST and IPSJ.

Takuo WATANABE is an Associate Professor of JAIST; he joined the faculty in 1992. He received his Ph.D. degree from Tokyo Institute of Technology in 1991. From 1990 to 1992, he worked at the University of Tokyo and the University Illinois at Urbana-Champaign as a JSPS junior researcher. His research interests include computational reflection, meta-level architecture, object-oriented computing, distributed computing and mobile/nomadic computing. He is a member of ACM, JSSST and IPSJ. 